\title{
Emotionally-Relevant Features for Classification and Regression of Music Lyrics
}

\author{
Ricardo Malheiro, Renato Panda, Paulo Gomes and Rui Pedro Paiva
}

\begin{abstract}
This research addresses the role of lyrics in the music emotion recognition process. Our approach is based on several state of the art features complemented by novel stylistic, structural and semantic features. To evaluate our approach, we created a ground truth dataset containing 180 song lyrics, according to Russell's emotion model. We conduct four types of experiments: regression and classification by quadrant, arousal and valence categories. Comparing to the state of the art features (ngrams - baseline), adding other features, including novel features, improved the F-measure from $69.9 \%, 82.7 \%$ and $85.6 \%$ to $80.1 \%, 88.3 \%$ and $90 \%$, respectively for the three classification experiments. To study the relation between features and emotions (quadrants) we performed experiments to identify the best features that allow to describe and discriminate each quadrant. To further validate these experiments, we built a validation set comprising 771 lyrics extracted from the AllMusic platform, having achieved $73.6 \%$ F-measure in the classification by quadrants. We also conducted experiments to identify interpretable rules that show the relation between features and emotions and the relation among features. Regarding regression, results show that, comparing to similar studies for audio, we achieve a similar performance for arousal and a much better performance for valence.
\end{abstract}

Index Terms-affective computing, affective computing applications, music retrieval and generation, natural language processing, recognition of group emotion

\section{INTRODUCTION}

$\mathrm{M}$ usic emotion recognition (MER) is gaining significant attention in the Music Information Retrieval (MIR) scientific community. In fact, the search of music through emotions is one of the main criteria utilized by users [1].

Real-world music databases from sites like AllMusic ${ }^{1}$ or Last.fm² ${ }^{2}$ grow larger and larger on a daily basis, which requires a tremendous amount of manual work for keeping them updated. Unfortunately, manually annotating music with emotion tags is normally a subjective process and an expensive and timeconsuming task. This should be overcome with the use of automatic recognition systems [2].

Most of the early-stage automatic MER systems were based on audio content analysis (e.g., [3]). Later on, researchers started combining audio and lyrics, leading to bi-modal MER systems with improved accuracy (e.g., [2], [4] [5]). This does not come as a surprise since it is evident that the importance of each dimension (audio or lyrics) depends on music style. For example, in dance music audio is the most relevant dimension, while in poetic music (like Jacques Brel) lyrics are key.

Several psychological studies confirm the importance of lyrics to convey semantical information. Namely, according to Juslin and Laukka [6], 29\% of people mention that lyrics are an important factor of how music expresses emotions. Also, Besson et al. [7] have shown that part of the semantic information of songs resides exclusively in the lyrics.

Despite the recognized importance of lyrics, current research in Lyrics-based MER (LMER) is facing the so-called glass-ceiling

- R. Malheiro is with Center for Informatics and Systems of the University of Coimbra (CISUC) and Miguel Torga Higher Institute.E-mail: rsmal@ dei.uc.pt.

- R. Panda, P. Gomes and R. P. Paiva are with Center for Informatics and Systems of the University of Coimbra (CISUC). E-mail: \{panda, pgomes, ruipedro\}@dei.uc.pt.
[8] effect (which also happened in audio). In our view, this ceiling can be broken with recourse to dedicated emotion-related lyrical features. In fact, so far most of the employed features are directly imported from general text mining tasks, e.g., bag-ofwords (BOW) and part-of-speech (POS) tags, and, thus, are not specialized to the emotion recognition context. Namely, these state-of-the-art features do not account for specific text emotion attributes, e.g., how formal or informal the text language is, how the lyric is structured and so forth.

To fill this gap we propose novel features, namely:

- Slang presence, which counts the number of slang words from a dictionary of 17700 words;

- Structural analysis features, e.g., the number of repetitions of the title and chorus, the relative position of verses and chorus in the lyric;

- Semantic features, e.g., gazetteers personalized to the employed emotion categories.

Additionally, we create a new, manually annotated, (partially) public dataset to validate the proposed features. This might be relevant for future system benchmarking, since none of the current datasets in the literature is public (e.g., [5]). Moreover, to the best of our knowledge, there are no emotion lyrics datasets in the English language that are annotated with continuous arousal and valence values.

The paper is organized as follows. In section 2, the related work is described and discussed. Section 3 presents the methods employed in this work, particularly the proposed features and ground truth. The results attained by our system are presented and discussed in Section 4. Finally, section 5 summarizes the main conclusions of this work and possible directions for future research. 


\section{ReLATEd Work}

The relations between emotions and music have been a subject of active research in music psychology for many years. Different emotion paradigms (e.g., categorical or dimensional) and taxonomies (e.g., Hevner, Russell) have been defined [9], [10] and exploited in different computational MER systems.

Identification of musical emotions from lyrics is still in an embryonic stage. Most of the previous studies related to this subject used general text instead of lyrics, polarity detection instead of emotion detection. More recently, LMER has gained significant attention by the MIR scientific community.

Feature extraction is one of the key stages of the LMER process. Previous works employing lyrics as a dimension for MER typically resort to content-based features (CBF) like Bag-OfWords (BOW) [5], [11], [12] with possible transformations like stemming and stopwords removal. Other regularly used CBFs are Part-Of-Speech (POS) followed by BOW [12]. Additionally, linguistic and text stylistic features [2], are also employed.

Despite the relevance of such features and their possibility of use in general contexts, we believe they do not capture several aspects that are specific of emotion recognition in lyrics. Therefore, we propose new features, as will be described in Section 3.

As for ground truth construction, different authors typically construct their own datasets, annotating the datasets either manually (e.g., [11]), or acquiring annotated data from sites such as AllMusic or Last.fm (e.g., [12], [13]).

As for systems based on manual annotations, it is difficult to compare them, since they all use different emotion taxonomies and datasets. Moreover, the employed datasets are not public. As for automatic approaches, frameworks like AllMusic or Last.fm are often employed. However, the quality of these annotations might be questionable because, for example in Last.fm, the tags are assigned by online users, which in some cases may cause ambiguity. In AllMusic, despite the fact that the annotations are made by experts [14], it is not clear whether they are annotating songs using only audio, lyrics or a combination of both.

Due to the limitations of the annotations in approaches like AllMusic and Last.fm and the fact that the datasets proposed by other researchers are not public, we decided to construct a manually annotated dataset. Our goal is to study the importance of each feature to the lyrics in a context of emotion recognition. So, the annotators have been told explicitely to ignore the audio during the annotations to measure the impact of the lyrics in the emotions. In the same way some researchers of the audio's area ask annotators to ignore lyrics, when they want to evaluate models focused on audio [15]. This all independently of in the process of audition we may use both dimensions. In the future we intend to fuse both dimensions and make a bimodal analysis. Additionally, to facilitate future benchmarking, the constructed dataset will be made partially public ${ }^{3}$, i.e., we provide the names of the artists and the song titles, as well as valence and arousal values, but not the song lyrics, due to copyright issues; instead we provide the URLs from where each lyric was retrieved.

Most current LMER approaches are black-box models instead of interpretable models. In [14], the authors use a human-comprehensible model to find out relations between features from General Inquirer (GI) and emotions. We use interpretable rules to match emotions and features not only from GI but from other types (e.g. Stylistic, Structural and Semantic) and platforms such as LIWC, ConcepNet and Synesketch.

\section{Methods}

\subsection{Dataset Construction}

As abovementioned, current MER systems either follow the categorical or the dimensional emotion paradigm. It is often argued that dimensional paradigms lead to lower ambiguity, since instead of having a discrete set of emotion adjectives, emotions are regarded as a continuum [11]. One of the most well-known dimensional models is Russell's circumplex model [16], where emotions are positioned in a two-dimensional plane comprising two axes, designated as valence and arousal, as illustrated in Figure 1. According to Russell [17], valence and arousal are the "core processes" of affect, forming the raw material or primitive of emotional experience.

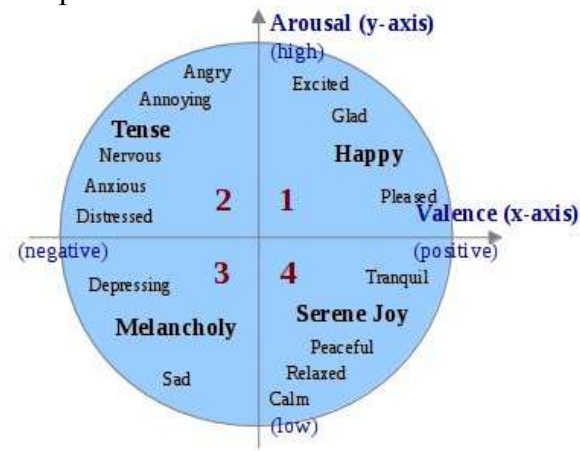

Figure 1. Russell's circumplex model (adapted from [11]).

\subsubsection{Data Collection}

To construct our ground truth, we started by collecting 200 song lyrics. The criteria for selecting the songs were the following:

- Several musical genres and eras (see Table 1);

- Songs distributed uniformly by the 4 quadrants of the Russell emotion model;

- Each song belonging predominantly to one of the 4 quadrants in the Russell plane.

To this end, before performing the annotation study described in the next section, the songs were pre-annotated by our team and were nearly balanced across quadrants.

Next, we used the Google API to search for the song lyrics. In this process, three sites were used for lyrical information: lyrics.com, ChartLyrics and MaxiLyrics.

The obtained lyrics were then preprocessed to improve their quality. Namely, we performed the following tasks:

- Correction of orthographic errors;

- Elimination of songs with non-English lyrics;

- Elimination of songs with lyrics with less than 100 characters;

- Elimination of text not related with the lyric (e.g., names of the artists, composers, instruments).

- Elimination of common patterns in lyrics such as [Chorus $\mathrm{x} 2$ ], [Vers1 x2], etc;

- Complementation of the lyric according to the corresponding audio (e.g., chorus repetitions in the audio are 
added to the lyrics).

To further validate our system, we have also built a larger validation set. This dataset was built in the following way:

1. First, we mapped the mood tags from AllMusic into the words from the ANEW dictionary (ANEW has 1034 words with values for arousal (A) and valence (V)). Depending on the values of $\mathrm{A}$ and $\mathrm{V}$, we can associate each word to a single Russell's quadrant. So, from that mapping, we obtained 33 words for quadrant 1 (e.g., fun, happy, triumphant), 29 words for quadrant 2 (e.g., tense, nervous, hostile), 12 words for quadrant 3 (e.g., lonely, sad, dark) and 18 words for quadrant 4 (e.g., relaxed, gentle, quiet).

2. Then, we considered that a song belongs to a specific quadrant if all of the corresponding AllMusic tags belong to this quadrant. Based on this requirement, we initially extracted 400 lyrics from each quadrant (the ones with a higher number of emotion tags), using the AllMusic's web service.

3. Next, we developed tools to automatically search for the lyrics files of the previous songs. We used 3 sites: Lyrics.com, ChartLyrics and MaxiLyrics.

4. Finally, this initial set was validated by three people. Here, we followed the same procedure employed by Laurier [5]: a song is validated into a specific quadrant if at least one of the annotators agreed with AllMusic's annotation (Last.FM in his case). This resulted into a dataset with 771 lyrics (211 for Q1, 205 for Q2, 205 for Q3, 150 for Q4). Even though the number of lyrics in Q4 is smaller, the dataset is still nearly balanced.

\subsubsection{Annotations and Validation}

The annotation of the dataset was performed by 39 people with different backgrounds. To better understand their background, we delivered a questionnaire, which was answered by $62 \%$ of the volunteers. $24 \%$ of the annotators who answered the questionnaire have musical training and, regarding their education level, $35 \%$ have a BSc degree, $43 \%$ have an MSc, $18 \%$ a $\mathrm{PhD}$ and $4 \%$ have no higher-education degree. Regarding gender balance, $60 \%$ were male and $40 \%$ were female subjects.

During the process, we recommended the following annotation methodology:

1. Read the lyric;

2. Identify the basic predominant emotion expressed by the lyric (if the user thought that there was more than one emotion, he/she should pick the predominant);

3. Assign values (between -4 and 4 ) to valence and arousal; the granularity of the annotation is the unit, which means that annotators could use 9 possible values to annotate the lyrics, from -4 to 4 ;

4. Fine tune the values assigned in 3) through ranking of the samples.

To further improve the quality of the annotations, the users were also recommended not to search for information about the lyric neither the song on the Internet or another place and to avoid tiredness by taking a break and continuing later.

We obtained an average of 8 annotations per lyric. Then, the arousal and valence of each song were obtained by the average of the annotations of all the subjects. In this case we considered the average trimmed by $10 \%$ to reduce the effect of outliers.

To improve the consistency of the ground truth, the standard deviation (SD) of the annotations made by different subjects for the same song was evaluated. Songs with an SD above 1.2 were excluded from the original set. As a result, 20 songs were discarded, leading to a final dataset containing 180 lyrics. This leads to a $95 \%$ confidence interval [18] of about \pm 0.4 . We believe this is acceptable in our -4.0 to 4.0 annotation range. Finally the consistency of the ground truth was evaluated using Krippendorff's alpha [19], a measure of inter-coder agreement. This measure achieved, in the range -4 up to $4,0.87$ and 0.82 respectively for the dimensions valence and arousal. This is considered a strong agreement among the annotators.

One important issue to consider is how familiar are the lyrics to the listeners. $13 \%$ of the respondents reported that they were familiar with $12 \%$ of the lyrics (on average). Nevertheless, it seems that the annotation process was sufficiently robust regarding the familiarity issue, since there was an average of 8 annotations per lyric and the annotation agreement (Krippendorff's alpha) was very high (as discussed in the following chapters). This suggests that the results were not skewed.

Although the size of the dataset is not large, we think that is acceptable for experiments and is similar to other datasets manually annotated (e.g., [11] has 195 songs).

Figures 2 and 3 show the histogram for arousal and valence dimensions as well as the distribution of the 180 selected songs for the 4 quadrants.
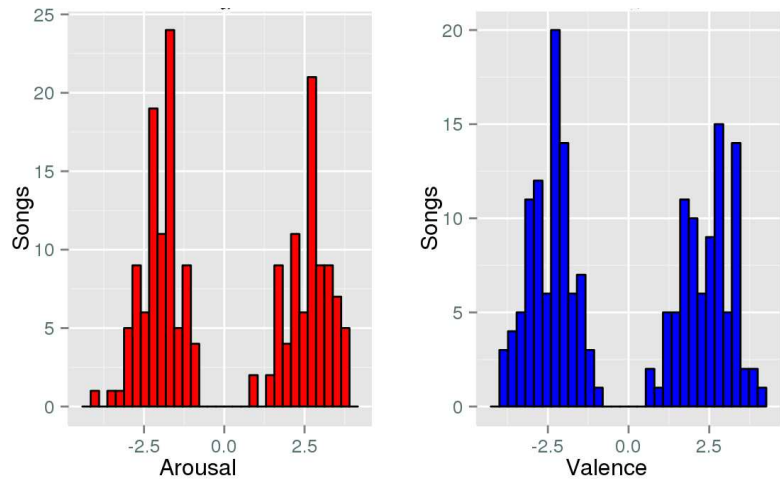

Figure 2. Arousal and valence histogram values.

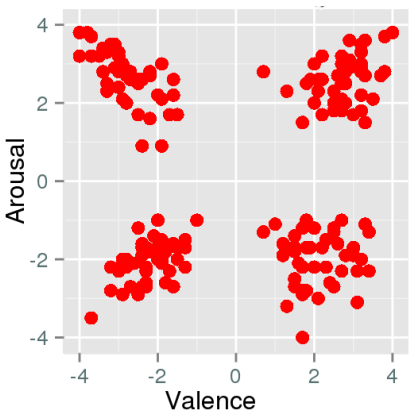

Figure 3. Distribution of the songs for the 4 quadrants.

Finally, the distribution of lyrics across quadrants and genres is presented in Table 1 . We can see that, except for quadrant 2 where almost half of the songs belong to the heavy metal genre, the other quadrants span several genres. 
Table 1. Distribution of lyrics across quadrants and genres.

\begin{tabular}{|l|c|c|c|c|}
\hline \multicolumn{1}{|c|}{ Genre } & Q1 & Q2 & Q3 & Q4 \\
\hline Pop/Rock & 6 & 1 & 15 & 11 \\
\hline Rock & 5 & 13 & 13 & 1 \\
\hline Heavy-metal & 0 & 20 & 1 & 0 \\
\hline Pop & 1 & 0 & 10 & 6 \\
\hline Jazz & 2 & 0 & 3 & 11 \\
\hline R\&B & 12 & 0 & 4 & 0 \\
\hline Dance & 16 & 0 & 0 & 0 \\
\hline New-age & 0 & 0 & 1 & 14 \\
\hline Hip-hop & 0 & 7 & 0 & 0 \\
\hline Country & 1 & 0 & 4 & 1 \\
\hline Reggae & 1 & 0 & 0 & 0 \\
\hline Total by Quadrant & 44 & 41 & 51 & 44 \\
\hline
\end{tabular}

\subsubsection{Emotion Categories}

Finally, each song is labeled as belonging to one of the four possible quadrants, as well as the respective arousal hemisphere (north or south) and valence meridian (east or west). In this work, we evaluate the classification capabilities of our system in the three described problems.

According to quadrants, the songs are distributed in the following way: quadrant 1 - 44 lyrics; quadrant 2 - 41 lyrics; quadrant 3 - 51 lyrics; quadrant $4-44$ lyrics (see Table 1 ).

As for arousal hemispheres, we ended up with 85 lyrics with positive arousal and 95 with negative arousal.

Regarding valence meridian we have 88 lyrics with positive valence positive and 92 with negative valence.

\subsection{Feature Extraction}

\subsection{Content-Based Features (CBF)}

The most commonly used features in text analysis, as well as in lyric analysis, are content-based features (CBF), namely the bagof-words (BOW) [20].

In this model the text in question is represented as a set of bags which normally correspond, in most cases, to unigrams, bigrams or trigrams. The BOW are normally associated to a set of transformations such as stemming and stopwords removal which are applied immediately after the tokenization of the original text. Stemming allows each word to be reduced to its stem and it is assumed that there are no differences, from the semantic point of view, in words which share the same stem. Through stemming the words "argue", "argued", "argues", "arguing" e "argus" would be reduced to the same stem "argu". The stopwords (e.g., the, is, in, at) which may also be called as function words are very common words in a certain language. These words bring normally little knowledge. The words include mainly determiners, pronouns and other gramatical particles which, by their frequency in a large quantity of documents, are not discriminative. The BOW may also be applied without any of the prior transformations. This technique was used, for example, in [12].

Part-of-speech (POS) tags are another type of state-of-art features. They consist in attributing a corresponding grammatical class to each word. For example the grammatical tagging of the following sentence "The student read the book" would be
"The/DT student/NN read/VBZ the/DT book/NN", where DT, NN and VBZ mean respectively determiner, noun and verb in 3rd person singular present. The POS tagging is typically followed by a BOW analysis. This technique was used in studies such as [21].

In our research we use all the combinations of unigrams, bigrams, trigrams with the aforementioned transformations. We also use n-grams of POS tags from bigram to 5-grams.

\subsubsection{Stylistic-Based Features (StyBF)}

These features are related to stylistic aspects of the language. One of the issues related to the written style is the choice of the type of the words to convey a certain idea (or emotion, in our study). Concerning music, those issues can be related to the style of the composer, the musical genre or the emotions that we intend to convey.

We use 36 features representing the number of occurrences of 36 different grammatical classes in the lyrics. We use the POS tags in the Penn Treebank Project [22] such as for instance JJ (adjectives), NNS (noum plural), RB (adverb), UH (interjection), VB (verb). Some of these features are also used by authors like [12].

We use two features related to the use of capital letters: All Capital Letters $(A C L)$, which represents the number of words with all letters in uppercase and First Capital Letter $(F C L)$, which represents the number of words initialized by an uppercase letter, excluding the first word of each line.

Finally, we propose a new feature: the number of occurrences of slang words (abbreviated as \#Slang). These slang words (17700 words) are taken from the Online Slang Dictionary ${ }^{4}$ (American, English and Urban Slang). We propose this feature because, in specific genres like hip-hop, the ideas are expressed normally with a lot of slang, so we believe that this feature may be important to describe specific emotions associated to specific genres.

\subsubsection{Song-Structure-Based Features (StruBF)}

To the best of our knowledge, no previous work on LMER employs features related to the structure of the lyric. However, we believe this type of features has relevance for LMER. Hence, we propose novel features of this kind, namely:

- \#CH, which stands for the number of times the chorus is repeated in the lyric;

- \#Title, which is the number of times the title appears in the lyric.

- 10 features based on the lyrical structure in verses (V) and chorus $(\mathrm{C})$ :

- \#VorC (total of sections - verses and chorus - in the lyrics);

○ \#V (number of verses);

- C... (the lyric starts with chorus - boolean);

- \#V/Total (relation between Vs and the total of sections);

- \#C/Total (relation between $\mathrm{C}$ and the total of sections);

- >2CAtTheEnd (lyric ends with at least two repetitions of the chorus - boolean);

- (3 features) alternation between versus and chorus, e.g., $V C V C$... (verses and chorus are alternated), VCCVCC... (between 2 verses we have at least 1 
chorus), $V V C V C$ (between 2 chorus we have at least 1 verse).

Common sense says, for example, that normally more danceable songs have more repetitions of the chorus. We believe that the different structures that a lyric may have, are taken into account by the composers to express emotions. That is the reason why we propose these features.

\subsubsection{Semantic-Based Features (SemBF)}

These features are related to semantic aspects of the lyrics. In this case, we used features based on existing frameworks like Synesketch ${ }^{5}$ (8 features), ConceptNet ${ }^{6}$ (8 features), LIWC $^{7}$ (82 features) and $\mathrm{GI}^{8}$ (182 features).

In addition to the previous frameworks, we use features based on known dictionaries: DAL [23] and ANEW [24]. From DAL (Dictionary of Affect in Language) we extract 3 features which are the average in lyrics of the dimensions pleasantness, activation and imagery. Each word in DAL is annotated with these 3 dimensions. As for ANEW (Affective Norms for English Words) we extract 3 features which are the average in lyrics of the dimensions valence, arousal and dominance. Each word in ANEW is annotated with these 3 dimensions.

Additionally, we propose 14 new features based on gazetteers, which represent the 4 quadrants of the Russell emotion model. We constructed the gazetteers according to the following procedure:

1. We define as seed words the 18 emotion terms defined in Russell's plane (see figure 1 in the article).

2. From the 18 terms, we consider for the gazetteers only the ones present in the DAL or the ANEW dictionaries. In DAL, we assume that pleasantness corresponds to valence, and activation to arousal, based on [25]. We employ the scale defined in Dal: arousal and valence (AV) values from 1 to 3 . If the words are not in the DAL dictionary but are present in ANEW, we still consider the words and convert the arousal and valence values from the ANEW scale to the DAL scale.

3. We then extend the seed words through Wordnet Affect [26], where we collect the emotional synonyms of the seed words (e.g., some synonyms of joy are exuberance, happiness, bonheur and gladness). The process of assigning the AV values from DAL (or ANEW) to these new words is performed as described in step 2 .

4. Finally, we search for synonyms of the gazetteer's current words in Wordnet and we repeat the process described in step 2.

Before the insertion of any word in the gazetteer (from step 1 on), each new proposed word is validated or not by two persons, according to its emotional value. There should be unanimity between the two annotators. The two persons involved in the validation were not linguistic scholars but were sufficiently knowledgeable for the task.

Table 2 illustrates some of the words for each quadrant.
Table 2. Examples of words from the gazetteers in each quadrant.

\begin{tabular}{|l|l|l|}
\hline \multicolumn{1}{|c|}{ Q1 } & \multicolumn{1}{c|}{ V } & \multicolumn{1}{c|}{ A } \\
\hline Dance & 2.29 & 2.3 \\
\hline Excited & 2.5 & 2.91 \\
\hline Fun & 2.84 & 2.56 \\
\hline Glad & 2.75 & 2.5 \\
\hline Joy & 2.88 & 2.31 \\
\hline
\end{tabular}

\begin{tabular}{|l|l|l|}
\hline \multicolumn{1}{|c|}{ Q2 } & \multicolumn{1}{c|}{ V } & \multicolumn{1}{c|}{ A } \\
\hline Afraid & 1.25 & 2.42 \\
\hline Agony & 1.36 & 2.27 \\
\hline Anger & 1 & 2.89 \\
\hline Anxiety & 1 & 2.8 \\
\hline Distressed & 1.24 & 2.35 \\
\hline
\end{tabular}

\begin{tabular}{|l|l|c|}
\hline \multicolumn{1}{|c|}{ Q3 } & \multicolumn{1}{c|}{ V } & \multicolumn{1}{c|}{ A } \\
\hline Depressed & 1.55 & 1.83 \\
\hline Gloom & 1.25 & 1.38 \\
\hline Lonely & 1 & 1.27 \\
\hline Sad & 1.38 & 1.43 \\
\hline Sorrow & 1.2 & 1.77 \\
\hline
\end{tabular}

\begin{tabular}{|l|l|c|}
\hline \multicolumn{1}{|c|}{ Q4 } & \multicolumn{1}{c|}{ V } & \multicolumn{1}{c|}{ A } \\
\hline Comfort & 3 & 1.33 \\
\hline Cozy & 2.6 & 1.58 \\
\hline Peace & 2.68 & 1.49 \\
\hline Relaxed & 2.5 & 1.35 \\
\hline Serene & 2.6 & 1.22 \\
\hline
\end{tabular}

Overall, the resulting gazeteers comprised 132, 214, 78 and 93 words respectively for the quadrants 1, 2, 3 and 4 .

The features extracted are:

- VinGAZQ1 (average valence of the words present in the lyrics that are also present in the gazetteer of the quadrant 1);

- AinGAZQ1 (average arousal of the words present in the lyrics that are also present in the gazetteer of the quadrant 1);

- VinGAZQ2 (average valence of the words present in the lyrics that are also present in the gazetteer of the quadrant 2);

- AinGAZQ2 (average arousal of the words present in the lyrics that are also present in the gazetteer of the quadrant 2);

- $\operatorname{Vin} G A Z Q 3$ (average valence of the words present in the lyrics that are also present in the gazetteer of the quadrant 3);

- Ain GAZQ3 (average arousal of the words present in the lyrics that are also present in the gazetteer of the quadrant 3);

- VinGAZQ4 (average valence of the words present in the lyrics that are also present in the gazetteer of the quadrant 4);

- AinGAZQ4 (average arousal of the words present in the lyrics that are also present in the gazetteer of the quadrant 4);

- \#GAZQ1 (number of words of the gazetteer 1 that are present in the lyrics);

- \#GAZQ2 (number of words of the gazetteer 2 that are present in the lyrics);

- \#GAZQ3 (number of words of the gazetteer 3 that are present in the lyrics);

- \#GAZQ4 (number of words of the gazetteer 4 that are present in the lyrics);

- VinGAZQ1Q2Q3Q4 (average valence of the words present in the lyrics that are also present in the gazetteers of the quadrants $1,2,3,4)$;

- AinGAZQ1Q2Q3Q4 (average arousal of the words present in the lyrics that are also present in the gazetteers

\footnotetext{
${ }^{7}$ http:/ / www.liwc.net/

8 http://www.wjh.harvard.edu/ inquirer

${ }^{5}$ http:/ / synesketch.krcadinac.com/blog/

${ }^{6}$ http://web.media.mit.edu/ hugo/conceptnet/
} 
of the quadrants $1,2,3,4)$.

\subsubsection{Feature grouping}

The proposed features are organized into four different feature sets:

CBF. We define 10 feature sets of this type: 6 are BOW (1gram up to 3-grams) after tokenization with and without stemming (st) and stopwords removal (sw); 4 are BOW (2-grams up to 5-grams) after the application of a POS tagger without st and $\mathrm{sw}$. These BOW features are used as the baseline, since they are a reference in most studies [2], [27].

StyBF. We define 2 feature sets: the first corresponds to the number of occurrences of POS tags in the lyrics after the application of a POS tagger (a total of 36 different grammatical classes or tags); the second represents the number of slang words (\#Slang) and the features related to words in capital letters (ACL and $F C L$ ).

StruBF. We define one feature set with all the structural features.

SemBF. We define 4 feature sets: the first with the features from Synesketch and ConceptNet; the second with the features from LIWC; the third with the features from GI; and the last with the features from gazetteers, DAL and ANEW.

We use the term frequency and the term frequency inverse document frequency (tfidf) as representation values in the datasets.

\subsection{Classification and Regression}

For classification and regression, we use Support Vector Machines (SVM) [28], since, based on previous evaluations, this technique performed generally better than other methods. A polynomial kernel was employed and a grid parameter search was performed to tune the parameters of the algorithm. Feature selection and ranking with the ReliefF algorithm [29] were also performed in each feature set, in order to reduce the number of features. In addition, for the best features in each model, we analyzed the resulting feature probability density functions (pdf) to validate the feature selection that resulted from ReliefF, as described below.

For both classification and regression, results were validated with repeated stratified 10-fold cross validation [30] (with 10 repetitions) and the average obtained performance is reported.

Since we performed a very high number of experiments and each task uses different settings, it is not possible to present the employed parameters. We present, as an example, only the parameters for the validation dataset (771 lyrics) in section 4.2.1.

\section{Results ANd Discussion}

\subsection{Regression Analysis}

The regressors for arousal and valence were applied using the feature sets for the different types of features (e.g., SemBF). Then, after feature selection, ranking and reduction with the ReliefF algorithm, we created regressors for the combinations of the best feature sets.

To evaluate the performance of the regressors the coefficient of determination $R^{2}$ [31] was applied. This is a statistic that gives information about the goodness of fit of a model. This measure indicates how well data fit a statistic model. If value is 1 , the model perfectly fits the data. A negative value indicates that the model does not fit the data at all.
Suppose a dataset with $n$ values marked as $y_{1} \ldots y_{n}$ (known as $y_{i}$ ), each associated with a predicted value $f_{1} \ldots f_{n}$ (known as $\left.f_{i}\right) . \bar{y}$ is the mean of the observed data. $R^{2}$ is calculated as in (1).

$$
R^{2}=1-\frac{\sum_{i}\left(y_{i}-f_{i}\right)^{2}}{\sum_{i}\left(y_{i}-\bar{y}\right)^{2}}
$$

$R^{2}$ was computed separately for each dimension (arousal and valence).

The results were 0.59 (with 234 features) for arousal and 0.61 (with 340 features) for valence. The best results were achieved always with RBFKernel [32].

Yang [11] made an analogous study using a dataset with 195 songs (using only the audio). He achieved a $R^{2}$ score of 0.58 for arousal and 0.28 for valence. We can see that we obtained almost the same results for arousal ( $0.59 \mathrm{vs} 0.58$ ) and much better results for valence ( $0.61 \mathrm{vs} 0.28)$. Although direct comparison is not possible, these results suggest that lyrics analysis is likely to improve audio-only valence estimation. Thus, in the near future, we will evaluate a bi-modal analysis using both audio and lyrics.

In addition, we used the obtained arousal and valence regressors to perform regression-based classification (discussed below).

\subsection{Classification Analysis}

We conduct three types of experiments for each of the defined feature sets: i) classification by quadrant categories; ii) classification by arousal hemispheres; iii) and classification by valence meridians.

\subsubsection{Classification By Quadrant Emotion Categories}

We can see in the following table (see Table 3) the performance of the best models for each one of the features categories (e.g., CBF). For CBF, we considered for example the two best models (M11 and M12). The field \#Features-SelFeatures-FMeasure(\%) represents respectively the total of features, the number of selected features and the results accomplished via the F-measure metric after feature selection.

Table 3. Classification by Quadrants: Best F-measure results for model.

\begin{tabular}{|l|l|l|}
\hline Model ID & Description & $\begin{array}{l}\text { \#Features- } \\
\text { SelFeatures- } \\
\text { FMeasure( } \%)\end{array}$ \\
\hline M11(CBF) & BOW (unigrams) & $3567-200-70.1$ \\
\hline M12(CBF) & POS+BOW(trigrams) & $4687-700-64.5$ \\
\hline M21(StyBF) & \#POS_Tags & $34-20-51$ \\
\hline M22(StyBF) & \#Slang+ACL+FCL & $3-3-36.7$ \\
\hline M31(StruBF) & Structural Lyric Features & $12-11-34.7$ \\
\hline M41(SemBF) & LIWC & $82-39-71.1$ \\
\hline M42(SemBF) & Features based on gazeteers & $20-20-65.3$ \\
\hline M43(SemBF) & GI & $182-90-61.7$ \\
\hline
\end{tabular}

In the table above, M1x stands for models that employ CBF features, M2x represents models with StyBF features, M3x StruBF features and M4x SemBF features. The same code is employed in the tables in the following sections.

The model M41 is not significantly better comparing to M11, but is significantly better than the model M42 (at $\mathrm{p}<0.05$ ). As for statistical significance we use the Wilcoxon rank-sum test. 
As we can see, the two best results were achieved with features from the state-of-the-art, namely BOW and LIWC. The results were close to the novel semantic features in M42 (65.3\%). The results of the other novel features (M22 and M31) were not so good in comparison to the baseline at least when evaluated in isolation.

Table 4 shows the results of the combination the best models for each of the features categories. For example C1Q is the combination of the CBF's best models after feature selection, i.e., initially, for this category, we have 10 different models (see section 3.2.5). After feature selection, the models are combined (only the selected features) and the result is $\mathrm{C} 1 \mathrm{Q}$. Then $\mathrm{C} 1 \mathrm{Q}$ has 900 features and after feature selection we got a result of $69.9 \%$ for Fmeasure. The classification process is analogous for the other categories.

In Table 4, \#Features represents the total of features of the model, Selected Features is the number of selected features and Fmeasure represents the results accomplished via the F-measure metric.

Table 4: Classification by Quadrants: Combination of the best models by categories.

\begin{tabular}{|c|c|c|c|}
\hline Model ID & \#Features & $\begin{array}{c}\text { Selected } \\
\text { Features }\end{array}$ & $\begin{array}{c}\text { F-meas- } \\
\text { ure (\%) }\end{array}$ \\
\hline C1Q (CBF) & 900 & 812 & 69.9 \\
\hline C2Q (StyBF) & 23 & 20 & 52.9 \\
\hline C3Q (StruBF) & 11 & 11 & 34.7 \\
\hline C4Q (SemBF) & 163 & 39 & 76.2 \\
\hline $\begin{array}{c}\text { Mixed } \\
\text { C1Q+C2Q+C3Q+C4Q }\end{array}$ & 1006 & 609 & 80.1 \\
\hline
\end{tabular}

As we can see, the combination of the best models of BOW (baseline) keep the results close to the 70\% (model C1Q) with a high number of features selected (812). The results of the SemBF (C4Q) are significantly better since we obtain a better performance $(76.20 \%)$ with much less features (39). It seems that the novel features (M42) have an important role in the overall improvement of the SemBF since the overall results for this type of features is $76.20 \%$ and the best semantic model (LIWC) achieved $71.10 \%$.

The mixed classifier $(80.1 \%)$ is significantly better than the best classifiers by type of feature: C1Q, C2Q, C3Q and C4Q (at $p$ $<0.05$ ). These results show the importance of the new features for the overall results.

Additionally, we performed regression-based classification based on the above regression analysis. An F-measure of 76.1\% was achieved, which is close to the quadrant-based classification. Hence, training only two regressor models could be applied to both regression and classification problems with reasonable accuracy.

Finally, we trained the 180-lyrics dataset using the mixed $\mathrm{C} 1 \mathrm{Q}+\mathrm{C} 2 \mathrm{Q}+\mathrm{C} 3 \mathrm{Q}+\mathrm{C} 4 \mathrm{Q}$ features, and validated the resulting model using the new larger dataset (comprising 771 lyrics). We obtained $73.6 \%$ F-measure, which shows that our model, trained in the 180-lyrics dataset, generalizes reasonably well. The parameters used for the SVM classifier with polynomial kernel were 2 for the complexity parameter $(\mathrm{C})$ and 0.6 for the exponent value of the polynomial kernel.

\subsubsection{Classification by Arousal Hemispheres}

We perform the same study for the classification by arousal hemispheres. Table 5 shows the results attained by the best models for each feature set.

Table 5. Classification by Arousal Hemispheres: Best F-measure results for model.

\begin{tabular}{|l|l|l|}
\hline Model ID & Description & $\begin{array}{l}\text { \#Features- } \\
\text { SelFeatures- } \\
\text { Fmeasure(\%) }\end{array}$ \\
\hline M11(CBF) & BOW (unigrams) & $3567-404-79.9$ \\
\hline M12(CBF) & POS+BOW(trigrams) & $4687-506-83.9$ \\
\hline M13(CBF) & POS+BOW(bigrams) & $700-290-77.7$ \\
\hline M21(StyBF) & \#POS_Tags & $34-24-77$ \\
\hline M22(StyBF) & \#Slang+ACL+FCL & $3-2-71.3$ \\
\hline M31(StruBF) & Structural Lyric Features & $12-8-70.2$ \\
\hline M41(SemBF) & LIWC & $82-50-79.9$ \\
\hline M42(SemBF) & Features based on gazeteers & $20-8-79.8$ \\
\hline M43(SemBF) & GI & $182-79-78.8$ \\
\hline M44(SemBF) & SYN+CN & $16-8-63$ \\
\hline
\end{tabular}

The best results $(83.90 \%)$ are obtained for trigrams after POS (M12). This suggests that the way the sentences are constructed, from a syntactic point of view, can be an important indicator for the arousal hemispheres of the lyrics. The trigram $v b+p r p+n n$ is an example of an important feature for this problem (taken from the ranking of features of this model). In this trigram, " $v b$ " is a verb in the base form, "prp" is a preposition and " $\mathrm{nn}$ " is a noun.

The novel features in StruBF (M31) and StyBF (M22) achieved respectively $70.2 \%$ with 8 features and $71.30 \%$ with 2 features. These results are above some state-of-the-art features like the features in M44 and these results are accomplished with few features ( 2 and 8 respectively). The results of the novel features in M42 seem promising since they are close to the best model M12 and with similar values compared to known platforms like LIWC and GI and with less features (8 to 50 and 70 respectively for LIWC and GI).

The model M12 is significantly better than the other classifiers (at $\mathrm{p}<0.05)$.

Table 6 shows the combinations by feature sets and the combination of the combinations respectively.

Table 6. Classification by Arousal Hemispheres: Combination of the best models by categories.

\begin{tabular}{|c|c|c|c|}
\hline Model ID & $\begin{array}{c}\text { \#Fea- } \\
\text { tures }\end{array}$ & $\begin{array}{c}\text { Selected } \\
\text { Features }\end{array}$ & $\begin{array}{c}\text { F-meas- } \\
\text { ure (\%) }\end{array}$ \\
\hline C1A (CBF) & 1690 & 1098 & $\mathbf{8 2 . 7}$ \\
\hline C2A (StyBF) & 26 & 26 & $\mathbf{7 5 . 4}$ \\
\hline C3A (StruBF) & 8 & 8 & $\mathbf{7 0 . 2}$ \\
\hline C4A (SemBF) & 163 & 39 & $\mathbf{7 6 . 2}$ \\
\hline $\begin{array}{c}\text { Mixed } \\
\text { C1A+C2A+C3A+C4A }\end{array}$ & 1196 & 377 & $\mathbf{8 8 . 3}$ \\
\hline
\end{tabular}

Comparing to best state of the art features (BOW), the best results with the combinations were improved from $82.7 \%$ to $88.3 \%$. The mixed classifier $(88.3 \%)$ is significantly better than best classifiers by type of feature: C1A, C2A, C3A and C4A (at p $<0.05)$, showing again the key role of the novel features.

\subsubsection{Classification by Valence Meridians}

We perform the same study for the classification by valence meridian. The following table (Table 7) shows the results of the best 
models by type of features.

Table 7. Classification by Valence Meridians: Best F-measure results for model.

\begin{tabular}{|l|l|l|}
\hline Model ID & Description & $\begin{array}{l}\text { \#Features- } \\
\text { SelFeatures- } \\
\text { FMeasure }(\%)\end{array}$ \\
\hline M13(CBF) & POS+BOW(bigrams) & $700-100-73.9$ \\
\hline M14(CBF) & BOW (unigrams+stemming) & $2856-395-81.7$ \\
\hline M15(CBF) & BOW(bigrams - tfidf) & $18139-600-68.2$ \\
\hline M22(StyBF) & \#Slang+ACL+FCL & $3-3-50.9$ \\
\hline M23(StyBF) & \#POS_Tags - tfidf & $34-11-69.4$ \\
\hline M31(StruBF) & Structural Lyric Features & $12-4-58.1$ \\
\hline M41(SemBF) & LIWC & $82-15-83.9$ \\
\hline M42(SemBF) & Features based on gazeteers & $20-16-82.8$ \\
\hline M43(SemBF) & GI & $182-87-82.2$ \\
\hline
\end{tabular}

These results show the importance of the semantic features in general, since the semantic models (M41, M42, M43) are significantly better than the classifiers of the other types of features (at $\mathrm{p}<0.05)$. Features related with the positivity or negativity of the words such as VinDAL or posemo (positive words) have an important role to these results.

Table 8 shows the combinations by feature sets and the combination of the combinations respectively.

Table 8. Classification by Valence Meridians: Combination of the best models by category.

\begin{tabular}{|c|c|c|c|}
\hline Model ID & \#Features & $\begin{array}{c}\text { Selected } \\
\text { Features }\end{array}$ & $\begin{array}{c}\text { F-measure } \\
(\%)\end{array}$ \\
\hline C1V (CBF) & 1095 & 750 & $\mathbf{8 5 . 6}$ \\
\hline C2V (StyBF) & 14 & 11 & $\mathbf{7 1}$ \\
\hline C3V (StruBF) & 4 & 4 & $\mathbf{5 8 . 1}$ \\
\hline C4V (SemBF) & 39 & 6 & $\mathbf{8 6 . 7}$ \\
\hline $\begin{array}{c}\text { Mixed } \\
\text { C1V+C2V+C3V+C4V }\end{array}$ & 771 & 594 & $\mathbf{9 0}$ \\
\hline
\end{tabular}

In comparison to the previous studies (quadrants and arousal), these results are better in general. We can see this in the BOW experiments (baseline- $85.60 \%$ ) where we achieved a performance close to the best combination $(\mathrm{C} 4 \mathrm{~V})$. The best results are also in general achieved with less features as we can see in $\mathrm{C} 3 \mathrm{~V}$ and $\mathrm{C} 4 \mathrm{~V}$.

The mixed classifier (90\%) is significantly better than the best classifiers by type of feature: $\mathrm{C} 1 \mathrm{~V}, \mathrm{C} 2 \mathrm{~V}, \mathrm{C} 3 \mathrm{~V}$ and $\mathrm{C} 4 \mathrm{~V}$ (at $\mathrm{p}<$ 0.05).

\subsubsection{Binary Classification}

As a complement to the multiclass problem seen previously, we also evaluated a binary classification (BC) approach for each emotion category (e.g., quadrant 1). Negative examples of a category are lyrics that were not tagged with that category but were tagged with the other categories. For example (see Table 9) the $\mathrm{BC}$ in the quadrant 1 uses 88 examples, 44 positive examples and 44 negative examples. The latter 44 examples are equally distributed by the other quadrants.

The results in Table 9 were reached using 396, 442, 290 and 696 features, respectively for the four sets of emotions (quadrants).
Table 9 - F-measure values for BC.

\begin{tabular}{|l|c|c|}
\hline Sets of Emotions & \#lyrics & F-measure (\%) \\
\hline Quadrant 1 & 88 & 88.6 \\
\hline Quadrant 2 & 82 & 91.5 \\
\hline Quadrant 3 & 102 & 90.2 \\
\hline Quadrant 4 & 88 & 88.6 \\
\hline
\end{tabular}

The good performance of these classifiers, namely for quadrant 2 , indict that the prediction models can capture the most important features of these quadrants.

The analysis of the most important features by quadrant will be the starting point for the identification of the best features by sets of emotions or quadrants, as detailed in section 4.4.

\subsection{New Features: Comparison to Baseline}

Considering $\mathrm{CBF}$ as the baseline in this area, we though it would be important to assess the performance of the models created when we add to the baseline the new proposed features. The new proposed features are contained in three categories: StyBF (feature set M22), StruBF (feature set M31) e SemBF (feature set M42). Next, we created new models adding to $\mathrm{C}^{*}$ each one of the previous feature sets in the following way: $\mathrm{C} 1^{*}+\mathrm{M} 22$; $\mathrm{C} 1^{*}+\mathrm{M} 31 ; \mathrm{C} 1^{*}+\mathrm{M} 42 ; \mathrm{C} 1^{*}+\mathrm{M} 22+\mathrm{M} 31+\mathrm{M} 42$. In $\mathrm{C} 1^{*}{ }^{*},{ }^{\prime} \mathrm{C} 1^{\prime}$ denotes a feature set that contains the combination of the best ContentBased Features - baseline and ' 1 ' denotes CBF, as mentioned above; "*" denotes expansion notation, indicating the different experiments conducted: $\mathrm{Q}$ denotes classification by quadrants, A by arousal hemispheres and $\mathrm{V}$ by valence meridians. These models were created for each of the 3 classification problems seen in the previous section: Classification by quadrants (see Table 10); classification by arousal (see Table 11); classification by valence (see Table 12).

Table 10. Classification by quadrants (baseline + new features).

\begin{tabular}{|c|c|c|}
\hline Model ID & $\begin{array}{c}\text { Selected } \\
\text { Features }\end{array}$ & $\begin{array}{c}\text { F-measure } \\
(\%)\end{array}$ \\
\hline C1Q+M22 & 384 & 72.1 \\
\hline C1Q+M31 & 466 & 70.4 \\
\hline C1Q+M42 & 576 & 78.4 \\
\hline C1Q+M22+M31+M42 & 388 & 82.7 \\
\hline
\end{tabular}

The baseline model (C1Q) alone reached $69.9 \%$ with 812 features selected (Table 4). We improve the results with all the combinations but only the models C1Q+M42 and $\mathrm{C} 1 \mathrm{Q}+\mathrm{M} 22+\mathrm{M} 31+\mathrm{M} 42$ are significantly better than the baseline model (at $\mathrm{p}<0.05)$. However the model $\mathrm{C} 1 \mathrm{Q}+\mathrm{M} 22+\mathrm{M} 31+\mathrm{M} 42$ is significantly better (at $\mathrm{p}<0.05$ ) than the model $\mathrm{C} 1 \mathrm{Q}+\mathrm{M} 42$. This shows that the inclusion of StruBF and StyBF have improved overall results.

Table 11. Classification by arousal (baseline + new features).

\begin{tabular}{|c|c|c|}
\hline Model ID & $\begin{array}{c}\text { Selected } \\
\text { Features }\end{array}$ & $\begin{array}{c}\text { F-measure } \\
(\%)\end{array}$ \\
\hline C1A+M22 & 652 & 83.3 \\
\hline C1A+M31 & 373 & 83.3 \\
\hline C1A+M42 & 690 & 84.4 \\
\hline C1A+M22+M31+M42 & 1307 & 84.6 \\
\hline
\end{tabular}

The baseline model (C1A) alone reached an F-measure of 
$82.7 \%$ with 1098 features selected (Table 6). We improve the results with all the combinations but only the models $\mathrm{C} 1 \mathrm{~A}+\mathrm{M} 42$ and $\mathrm{C} 1 \mathrm{~A}+\mathrm{M} 22+\mathrm{M} 31+\mathrm{M} 42$ are significantly better than the baseline model (at $\mathrm{p}<0.05)$. The inclusion of the features from M22 and $\mathrm{M} 31$ in $\mathrm{C} 1 \mathrm{~A}+\mathrm{M} 22+\mathrm{M} 31+\mathrm{M} 42$ improved the performance in comparison to the model $\mathrm{C} 1 \mathrm{~A}+\mathrm{M} 42$, since $\mathrm{C} 1 \mathrm{~A}+\mathrm{M} 22+\mathrm{M} 31+$ M42 is significantly better than the model C1A+M42 (at p < 0.05).

Table 12. Classification by valence (baseline + new features).

\begin{tabular}{|c|c|c|}
\hline Model ID & $\begin{array}{c}\text { Selected } \\
\text { Features }\end{array}$ & $\begin{array}{c}\text { F-measure } \\
(\%)\end{array}$ \\
\hline C1V+M22 & 679 & 85 \\
\hline C1V+M31 & 659 & 83.9 \\
\hline C1V+M42 & 493 & 87.8 \\
\hline C1V+M22+M31+M42 & 88 & 88.3 \\
\hline
\end{tabular}

The baseline model $(\mathrm{C} 1 \mathrm{~V})$ alone reached an F-measure of $85.6 \%$ with 750 features selected (Table 8 ). We improve the results with all the combinations but only the models $\mathrm{C} 1 \mathrm{~V}+\mathrm{M} 42$ and $\mathrm{C} 1 \mathrm{~V}+\mathrm{M} 22+\mathrm{M} 31+\mathrm{M} 42$ are significantly better than the baseline model (at $\mathrm{p}<0.05)$, however $\mathrm{C} 1 \mathrm{~V}+\mathrm{M} 22+\mathrm{M} 31+\mathrm{M} 42$ is not significantly better than $\mathrm{C} 1 \mathrm{~V}+\mathrm{M} 42$. This suggests the importance of the SemBF for this task in comparison to the other new features.

In general, the new StyBF and StruBF are not good enough to improve significantly the baseline score, however we got the same results with much less features: for classification by quadrants we decrease the number of features of the model from 812 (baseline) to 384 (StyBF) and 466 (StruBF). The same happens for arousal classification (1098 features - baseline to 652 - StyBF and 373 - StruBF) and for valence classification (750 features - baseline to 679 - StyBF and 659 - StruBF).

However, the model with all the features is always better (except for valence classification) than the model with only baseline and SemBF. This shows a relative importance of the novel StyBF and StruBF. It is important to highlight that M22 has only 3 features and M31 has 12 features.

The new SemBF (model M42) seems important because it can improve clearly the score of the baseline. Particularly in the last problem (classification by valence) it requires a much less number of features (750 down to 88).

\subsection{Best Features by Classification Problem}

We determined in the previous section the classification models with best performance for the several classification problems. These models were built through the interaction of a set of features (from the total of features after feature selection). Some of these features are possibly strong to predict a class when they are alone but others are strong only when combined with other features.

Our purpose in this section is to identify the most important features, when they act alone, for the description and discrimination of the problem's classes.

We will determine the best features for:

- Arousal (Hemispheres) description - the classes used are negative arousal (AN) and positive arousal (AP)

- Valence (Meridians) description - negative valence (VN) and positive valence (VP)

- Arousal when valence is positive - negative arousal (AN) and positive arousal (AP), which means quadrant 1 vs quadrant 4
- Arousal when valence is negative - negative arousal (AN) and positive arousal (AP), which means quadrant 2 vs quadrant 3

- Valence when arousal is positive - negative valence $(\mathrm{VN})$ and positive valence (VP), which means quadrant 1 vs quadrant 2

- Valence when arousal is negative - negative valence $(\mathrm{VN})$ and positive valence (VP), which means quadrant 3 vs quadrant 4

In all the situations we identify the 5 features that, after analysis, seem the best features. This analysis starts from the rankings (top 20) of the best features extracted from the models of the section 4.2, with ReliefF. Next, to validate ReliefF's ranking, we compute the probability density functions (pdf) [31] for each of the classes of the previous problems. Through the analysis of these pdfs we take some conclusions about the description of the classes and identify some of their main characteristics.

The images below show the pdfs of 2 of the 5 best features for the problem of valence description when the arousal is positive (distinguish between $1^{\text {st }}$ quadrant and $2^{\text {nd }}$ quadrant) (Figure 4). The features are M44-Anger_Weight_Synesketch (a) and M42-Di$n A N E W(b)$.

(a)

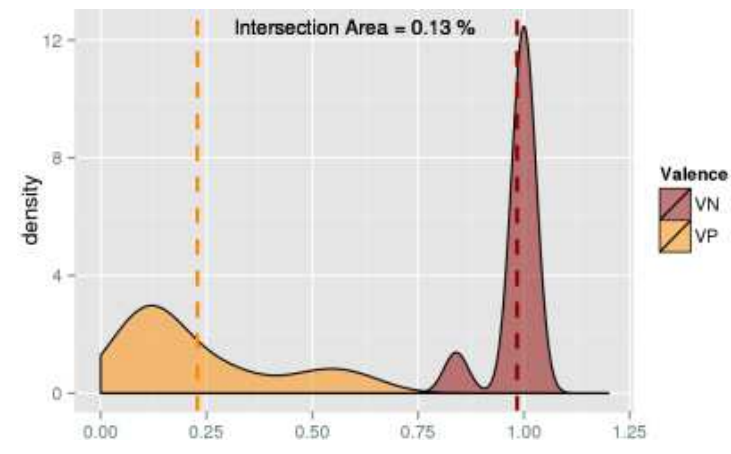

(b)

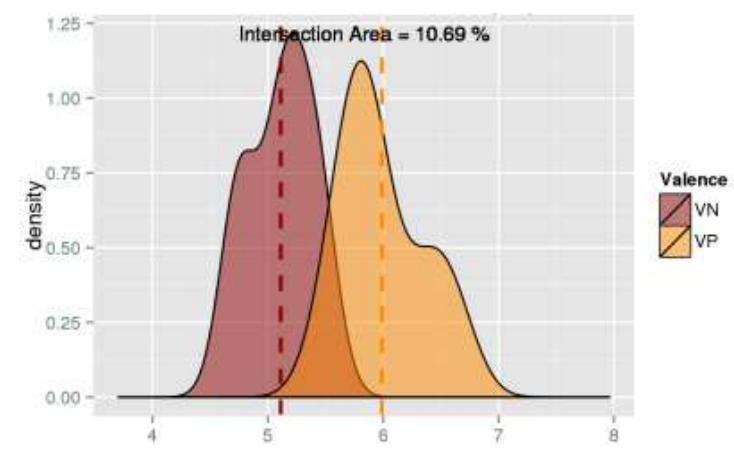

Figure 4. pdf of the features a) Anger_Weight_Synesketch and b) DinANEW for the problem of valence description when arousal is positive.

As we can see, the feature in the top image is more important for discriminating between the $1^{\text {st }}$ and $2^{\text {nd }}$ quadrants than the feature in the second image, because the density functions (f) are more separated. We use one measure (2) that indicates this separation: Intersection_Area, which represents the intersection area (in percentage) between the two functions. 


$$
\text { Intersection_Area }=\frac{\int f_{A} \cap \int f_{B}}{\int f_{A} \cup \int f_{B}}
$$

In (2), $\mathrm{A}$ and $\mathrm{B}$ are the compared classes (VN and VP in the example of the Figure 4 ) and $f_{A}$ and $f_{B}$ are respectively the pdfs for $\mathrm{A}$ and $\mathrm{B}$.

For this measure, lower values indicate more separation between the curves.

Both features are important to describe the quadrants. The first, taken from the Synesketch framework measures the weight of anger in the lyrics and, as we can see, it has higher values for the $2^{\text {nd }}$ quadrant as expected, since anger is a typical emotion from the $2^{\text {nd }}$ quadrant. The $2^{\text {nd }}$ feature represents the average dominance of the ANEW's words in the lyrics and, although some overlap, it shows that predominantly higher values indicate the $1^{\text {st }}$ quadrant and lower values indicate the $2^{\text {nd }}$ quadrant.

Based on above metric, the top- 5 best features were identified for each problem, i.e., the features that separate better the different problems.

\subsubsection{Best Features for Arousal Description}

As we can see (Table 13), the two best features to discriminate between arousal hemispheres are new features proposed by us. FCL represents the number of words started by a capital letter and it describes better the class AP than the class AN, i.e., lyrics with FCL greater than a specific value correspond normally to lyrics from the class AP. For low values there is a mix between the 2 classes. The same happens to \#Slang, \#Title, WC (word count - LIWC), active (words with active orientation - GI) and $v b$ (number of verbs in the base form). The feature negate (number of negations - LIWC) has an opposite behavior, i.e., mix between classes for lower values and the class $\mathrm{AN}$ from a specific point. The features not listed above, sad (words of the negative emotion sadness - LIWC), angry (angry weight in ConcepNet) and numb (words indicating the assessment of quantity, including the use of numbers - GI) have a similar pattern of behavior as the feature negate, while the novel features $\mathrm{CH}$ (number of repetitions of the chorus) and TotalVorCH (number of repetitions of verses or chorus) have similar pattern of behavior as the feature FCL.

Table 13. Best features for arousal description (classes AN, AP).

\begin{tabular}{|l|c|}
\hline \multicolumn{1}{|c|}{ Feature } & Intersection Area \\
\hline M22-FCL & $24.6 \%$ \\
\hline M22-\#Slang & $29 \%$ \\
\hline M43- active & $33.1 \%$ \\
\hline M21- vb & $34.2 \%$ \\
\hline M31-\#Title & $37.4 \%$ \\
\hline
\end{tabular}

\subsubsection{Best Features for Valence Description}

The best features and not only the 5 on Table 14 , are essentially semantic features. The feature $\operatorname{VinD} A L$ can describe both classes: lower values are more associated to the class $\mathrm{VN}$ and higher values to the class VP. The feature DinANEW has a similar pattern but not so good. The features VinGAZQ1Q2Q3Q4, negemo (words associated with negative emotions - LIWC), negativ (words of negative outlook - GI) and VinANEW are better for discrimination of the VN class. For the VP class they are not so good. The feature posemo (number of positive words - LIWC) for example describes better the VP class.
Table 14. Best features for valence description (classes VN, VP).

\begin{tabular}{|l|c|}
\hline \multicolumn{1}{|c|}{ Feature } & Intersection Area \\
\hline M41- posemo & $18.5 \%$ \\
\hline M43- negativ & $24.8 \%$ \\
\hline M42-VinDAL & $25.6 \%$ \\
\hline M42-VinGAZQ1Q2Q3Q4 & $25.8 \%$ \\
\hline M42- VinANEW & $26.1 \%$ \\
\hline
\end{tabular}

\subsubsection{Best Features For Arousal when Valence is Positive.}

As can be seen in Table 15, the features \#GAZQ1, FCL, iav (verbs giving an interpretative explanation of an action - GI), motion (measures dimension motion - LIWC), $v b$ (verbs in base form, $v b n$ (verbs in past participle), active, you (pronouns indicating another person is being addressed directly - GI) and \#Slang are good for discrimination of the $1^{\text {st }}$ quadrant (higher values associated to the class AP).

The features angry_CN, numb and article (number of articles LIWC) are good for discrimination of the $4^{\text {th }}$ quadrant. The feature Ain GAZQ1Q2Q3Q4 is good for both quadrants.

Table 15. Best features for arousal $(\mathrm{V}+)$ (classes AN, AP).

\begin{tabular}{|l|c|}
\hline \multicolumn{1}{|c|}{ Feature } & Intersection Area \\
\hline M42-\#GAZQ1 & $4.6 \%$ \\
\hline M43- active & $12.5 \%$ \\
\hline M21- vbn & $17.6 \%$ \\
\hline M43- you & $17.8 \%$ \\
\hline M21- vb & $18.7 \%$ \\
\hline
\end{tabular}

\subsubsection{Best Features for Arousal when Valence is Negative}

These features are summarized in Table 16. The features Anger_Weight_Synesketch and Disgust_Weight_Synesketch (weight of the emotion disgust) are good to discriminate between the quadrants 2 and 3 (higher values are associated as it was predictable to instances from the quadrant 2), although in the latter we have more overlap between the classes than in the prior. The features $v b p$ (verb, non-3rd person singular present) and anger can discriminate the class AP (higher values) but for lower values we have a mix between the classes. Other features with similar behavior are FCL, \#Slang, negativ (negative words - GI), cc (number of coordinating conjunctions) and \#Title. AinGAZQ2 and past can discriminate the $3^{\text {rd }}$ quadrant, i.e., the class AN. Finally the feature article (the number of definite, e.g., the, and indefinite, e.g., a, an, articles in the text) can discriminate both quadrants (tendency for $3^{\text {rd }}$ quadrant with lower values and $2^{\text {nd }}$ quadrant with higher values).

Table 16. Best features for arousal (V-) (classes AN, AP).

\begin{tabular}{|l|c|}
\hline \multicolumn{1}{|c|}{ Feature } & Intersection Area \\
\hline $\begin{array}{l}\text { M44-Anger_ } \\
\text { Weight_Synesketch }\end{array}$ & $7.9 \%$ \\
\hline M42-AinGAZQ2 & $16.2 \%$ \\
\hline M21-vbp & $17.8 \%$ \\
\hline M41-anger & $21.1 \%$ \\
\hline M21- cc & $25.4 \%$ \\
\hline
\end{tabular}




\subsubsection{Best Features for Valence when Arousal is Positive.}

The feature Anger_Weight_Synesketch is clearly discriminative to separate the quadrants 2 and 3 (see Table 17 and Figure 4). The novel semantic features VinANEW, VinGAZQ1Q2Q3Q4, VinDAL and DinANEW have a similar pattern behavior to the first feature but with a little overlap between the functions. The features negemo (negative emotion words - LIWC), swear (swear words - LIWC), negative (words of negative outlook - GI) and hostile (words indicating an attitude or concern with hostility or aggressiveness - GI) are good for the discrimination of the $2^{\text {nd }}$ quadrant (higher values).

Table 17. Best features for valence $(\mathrm{A}+$ ) (classes VN, VP).

\begin{tabular}{|l|c|}
\hline \multicolumn{1}{|c|}{ Feature } & Intersection Area \\
\hline $\begin{array}{l}\text { M44-Anger_Weight_Sy- } \\
\text { nesketch }\end{array}$ & $0.1 \%$ \\
\hline M42- VinANEW & $4.4 \%$ \\
\hline M42- VinGAZQ1Q2Q3Q4 & $7.2 \%$ \\
\hline M42- VinDAL & $7.7 \%$ \\
\hline M42- DinANEW & $10.7 \%$ \\
\hline
\end{tabular}

\subsubsection{Best Features for Valence when Arousal is Negative.}

The best features for valence discrimination when arousal is negative are presented in Table 18.

Between the quadrants 3 and 4, the features $v b d, I$, self and motion are better for the $3^{\text {rd }}$ quadrant discrimination, while the features \#GAZQ4, article, cc and posemo are better for $4^{\text {th }}$ quadrant discrimination.

Table 18. Best features for valence (A-) (classes VN, VP).

\begin{tabular}{|l|c|}
\hline \multicolumn{1}{|c|}{ Feature } & Intersection Area \\
\hline M41- posemo & $15.6 \%$ \\
\hline M43- self & $24.9 \%$ \\
\hline M21-vbd & $27 \%$ \\
\hline M42-\#GAZQ4 & $28.4 \%$ \\
\hline M41- motion & $29.2 \%$ \\
\hline
\end{tabular}

\subsubsection{Best Features by Quadrant}

Until now we have identified features important to discriminate, for example, between two quadrants. Next, we will evaluate if these features can discriminate completely the four quadrants, i.e., one quadrant against the other three.

To evaluate the quality of the discrimination of a specific feature concerning a quadrant $\mathrm{Qz}$, we have established a metric based on two measures:

- Discrimination support (support of a function is the set of points where the function is not zero-valued [33]), which corresponds to the difference between the total support of the two pdf (Qz and Qothers) and the support of the Qothers pdf, as defined in (3). The result is the support of the Qz pdf except the support of the intersection area and is in percentage of the total support. The higher this metric the better;

$$
\operatorname{Disc\_ sup}=\frac{\operatorname{len}\left(\sup \left(f_{Q_{Z}} \cup f_{Q_{\text {others }}}\right)\right)-\operatorname{len}\left(\sup \left(f_{Q_{\text {others }}}\right)\right)}{\operatorname{len}\left(\sup \left(f_{Q_{Z}} \cup f_{Q_{\text {others }}}\right)\right)}
$$

In (3), len(sup(f)) stands for the length of the support of function $f$ and $f_{Q_{Z}}$ and $f_{Q_{\text {others }}}$ are respectively the pdfs for Qz and
Qothers.

- Discrimination area, which corresponds to the difference between the area of the Qz's pdf and the intersection area between the two pdf, as in (4). The result is in percentage of the Qz's pdf total area. The higher this metric the better (Equation 4).

$$
\text { Disc_area }=\frac{\int f_{Q_{Z}}-\left(\int f_{Q_{Z}} \cap \int f_{Q_{\text {others }}}\right)}{\int f_{Q_{Z}}}
$$

In this analysis (Table 19), we have experimentally defined a minimum threshold of $30 \%$ for the Discrimination_Support. To do the ranking of the best features, we use the metric Discrimination_support and in case of a draw, we use the metric Discrimination_Area.

Table 19. Type of discrimination of the features by quadrant.

\begin{tabular}{|l|c|c|}
\hline \multicolumn{1}{|c|}{ Feature } & $\begin{array}{c}\text { Disc_Support / } \\
\text { Disc_Area (\%) }\end{array}$ & Quadrant \\
\hline M42_\#GAZQ1 & $75.4 / 66.3$ & Q1 \\
\hline M43_socrel & $62.4 / 29.5$ & Q1 \\
\hline M43_solve & $60.8 / 25.8$ & Q1 \\
\hline M41_humans & $59.1 / 28.6$ & Q1 \\
\hline M43_passive & $48.1 / 29.2$ & Q1 \\
\hline M31- \#Title & $41.1 / 36.2$ & Q1 \\
\hline M21- vbp & $40.3 / 32.8$ & Q1 \\
\hline M44_Happy_CN & $39.7 / 19.9$ & Q1 \\
\hline M44_CN-A & $30.1 / 22.1$ & Q1 \\
\hline M41-anger & $84.9 / 74$ & Q2 \\
\hline M21-vbg & $56 / 30.6$ & Q2 \\
\hline M43_negativ & $52.7 / 51.4$ & Q2 \\
\hline M22-\#Slang & $52.7 / 33.5$ & Q2 \\
\hline M41- negemo & $50.2 / 52$ & Q2 \\
\hline M21-nn & $49.7 / 31.5$ & Q2 \\
\hline M41-WC & $49.3 / 32.1$ & Q2 \\
\hline M43_wittot & $46.5 / 23.5$ & Q2 \\
\hline M22- FCL & $46.1 / 36.6$ & Q2 \\
\hline M21-dt & $45.7 / 31.2$ & Q2 \\
\hline M43-hostile & $45.2 / 45.6$ & Q2 \\
\hline M21-cc & $45.1 / 30.5$ & Q2 \\
\hline M21-prp & $40 / 36$ & Q2 \\
\hline M42-\#GAZQ3 & $63.3 / 41.3$ & Q3 \\
\hline M41-negate & $38.9 / 33.8$ & Q3 \\
\hline M41-cogmech & $32.9 / 19.9$ & Q3 \\
\hline M42-VinGAZQ1Q2Q3Q4 & $32.4 / 10.5$ & Q3 \\
\hline M42-\#GAZQ4 & $56.1 / 36.8$ & Q4 \\
\hline M41-Dic & $47.2 / 17.8$ & Q4 \\
\hline M41-hear & $46 / 19.5$ & Q4 \\
\hline M31-totalVorCH & $40.7 / 27.8$ & Q4 \\
\hline M42-DinDAL & $39.3 / 20.9$ & Q4 \\
\hline
\end{tabular}

Among the features that best represent each quadrant, we have features from the state of the art, such as features, from LIWC (M41) - humans (references to humans), anger (affect words), negemo (negative emotion words), WC (word count), negate (negations), cogmech (cognitive processes), Dic (dictionary words) and hear (hearing perceptual process); from GI (M43) - 
socrel (words for socially-defined interpersonal processes), solve (words referring to the mental processes associated with problem solving), passive (words indicating a passive orientation), negativ (negative words) and hostile (words indicating an attitude or concern with hostility or aggressiveness); from ConcepNet (M44) - happy_CN (happy weight), CN_A (arousal weight); from POS Tags (M21) - vbp (verb, non-3rd person singular present), vbg (verb, gerund or present participle), $n n$ (noun, singular or mass), $d t$ (determiner), $c c$ (coordinating conjunction) and prp (personal pronoun). We have also novel features, such as, StyBF (M22) - \#Slang and FCL; StruBF (M31) - \#Title and TotalVorCH; SemBF (M42) - \#GAZQ1, \#GAZQ3, VinGAZQ1Q2Q3Q4, \#GAZQ4 and DinDAL.

Some of the more salient characteristics of each of the quadrants:

- Q1: typically lyrics associated to songs with positive emotions and high activation. Songs from this quadrant are often associated to specific musical genres, such as, dance, pop and by the importance of the features we point out the features related with repetitions of the chorus and title in the lyric.

- Q2: we point out stylistic features such as \#Slang and FCL that indict high activation with predominance of negative emotions or features that are related with negative valence such as negativ (negative words), hostile (hostile words) and swear (swear words). This kind of features influence more Q2 than Q3 (although Q3 have also negative valence) because Q2 is more influenced by specific vocabulary such as the vocabulary in that features, while Q3 is more influenced by negative ideas, so we think that it is more difficult the perception of emotions in the $3^{\text {rd }}$ quadrant.

- Q3: we point out the importance of the verbal tense (past) in comparison with the other quadrants which have the predominance of the present tense. On the contrary, Q2 have also some tendency to the gerund tense and the Q1 to the present simple. We highlight also in comparison with the other quadrants more use of the $1^{\text {st }}$ singulier person (I).

- Q4: Features related with activation, as we have seen for the quadrants 1 and 2, have low weight for this quadrant. We point out the importance of a specific vocabulary as we have in \#GAZQ4.

Generally, semantic features are more important to discriminate the valence (e.g. VinDAL, VinANEW). Features important for sentiment analysis such as posemo (positive words) or ngtv (negative words) are also important for valence discrimination.

On the other hand, stylistic features related with the activation of the written text such as \#Slang or FCL are important for arousal discrimination. Features related with the weight of emotions in the written text are also important (e.g. $A n$ ger_Weight_Synesketch, Disgust_Weight_Synesketch).

\subsection{Interpretability}

After we have made a study to understand the best features to describe and discriminate each set of emotions, we are going to extract some rules/knowledge that allow to understand how these features and emotions are related. With this study we intend to attain two possible goals: i) find out relations between features and emotions (e.g., if feature $A$ is low and feature $B$ is high then the song lyrics belong to quadrant 2); ii) find out relations among features (e.g., song lyrics with feature A high also have feature B low).

\subsubsection{Relations between features and quadrants}

In this analysis we use the Apriori algorithm [34].

First, we pre-processed the employed features through the detection of features with a nearly uniform distribution, i.e., the feature values depart at most $10 \%$ from the feature mean value. We did not consider these kind of features. Here, we employed all the features selected in Mixed $\mathrm{C} 1 \mathrm{Q}+\mathrm{C} 2 \mathrm{Q}+\mathrm{C} 3 \mathrm{Q}+\mathrm{C} 4 \mathrm{Q}$ model (see Table 4), except for the ones excluded as described. In total, we employed 144 features.

Then we defined the following premises.

- Consideration of only rules up to 2 antecedents. It was applied an algorithm to eliminate redundance, considering the more generic rules to avoid complex rules;

- Due to the fact that n-grams features are sparse, we did not consider rules with part of the antecedent of type $\mathrm{n}$-gram $=$ Very Low. It means probably that the feature does not exist;

- Features were discretized in 5 classes using equal-frequency discretization: very low (VL), low (L), medium $(\mathrm{M})$, high $(\mathrm{H})$, very high $(\mathrm{VH})$. Rules containing nonuniform distributed features were ignored.

We considered two measures to assess the quality of the rules: confidence and support. The ideal rule has simultaneously high representativity (support) and high confidence degree.

Table 20 shows up the best rules for quadrants. We defined a threshold of support $=8.3 \%$ (15 lyrics) and confidence $=60 \%$.

We think this rules are in general self-explanatory and understandable, however we will explain some of them not so explicit.

We can see for Q1 the importance of the feature \#GAZQ1 together with the feature from GI, afftot (words in the affect domain), both with $\mathrm{VH}$ values. We can also highlight for this quadrant the relation between a VL weight for sadness and a VH value for the feature positiv (words of positive outlook) and the relation between a VH number of title's repetitions in the lyric and a VL weight for the emotion angry.

We can point out for quadrant 2 the importance of the features anger from LIWC and Synesketch, negemo_GI (negative emotion), \#GAZQ2, VinANEW, hostile (words indicating an attitude or concern with hostility or aggressiveness), powcon (words for ways of conflicting) and some combinations among them.

For quadrant 3 , we can point out the relation between a $\mathrm{VH}$ value for the emotion sadness and a VL value for the number of swear words in the lyrics.

For quadrant 4 we can point out the relation between the features anger and weak (words implying weakness) both with VL values.

These results confirm the results reached in the previous section, where we identified the most important features for each quadrant. 
Table 20. Rules from classification association mining.

\begin{tabular}{|c|c|c|}
\hline$\#$ & Rule & $\begin{array}{c}\text { Support/ } \\
\text { confidence } \\
(\%)\end{array}$ \\
\hline 1 & \#GAZQ1=VH ==> Q=Q1 & $13.8 / 80$ \\
\hline 2 & $\begin{array}{l}\text { \#GAZQ1=VH and afftot_GI=VH => } \\
\text { Q1 }\end{array}$ & $8.8 / 72$ \\
\hline 3 & $\begin{array}{l}\text { sad_LIWC=VL and positiv_GI=VH } \\
=>\text { Q1 }\end{array}$ & $7.7 / 82$ \\
\hline 4 & \#Title=VH and angry_CN=VL => Q1 & $7.2 / 72$ \\
\hline 5 & VinANEW $=\mathrm{VL}=>\mathrm{Q} 2$ & $20 / 61$ \\
\hline 6 & $\begin{array}{l}\text { hostile_GI=VH and Sad- } \\
\text { ness_Weight_Synesketch=VH => Q2 }\end{array}$ & $14.4 / 69$ \\
\hline 7 & $\begin{array}{l}\text { Anger_Weight_Synesketch }=\mathrm{VH} \text { and } \\
\text { Valence_Synesketch }=\mathrm{VL}=>\mathrm{Q} 2\end{array}$ & $12.7 / 76$ \\
\hline 8 & anger_LIWC $=\mathrm{H}=>\mathrm{Q} 2$ & $11.1 / 85$ \\
\hline 9 & negemo_GI=VH => Q2 & $11.1 / 67$ \\
\hline 10 & \#GAZQ2=VH => Q2 & $10.5 / 100$ \\
\hline 11 & $\begin{array}{l}\text { Anger_Weight_Synesketch=VH and } \\
\text { negemo_LIWC=VH => Q2 }\end{array}$ & $8.8 / 94$ \\
\hline 12 & anger_LIWC $=\mathrm{VH}=>\mathrm{Q} 2$ & $8.8 / 100$ \\
\hline 13 & VinGAZQ2=VH => Q2 & $8.3 / 83$ \\
\hline 14 & $\begin{array}{l}\text { hostile_GI=VH and powcon_GI=VH } \\
=>\mathrm{Q} 2\end{array}$ & $8.3 / 78$ \\
\hline 15 & $\begin{array}{l}\text { sad_LIWC }=\mathrm{VH} \text { and swear_LIWC }=\mathrm{VL} \\
=>\mathrm{Q} 3\end{array}$ & $8.8 / 72$ \\
\hline 16 & $\mathrm{dt}=\mathrm{VL}$ and article_LIWC $=\mathrm{VL}=>\mathrm{Q} 3$ & $8.3 / 71$ \\
\hline 17 & $\begin{array}{l}\mathrm{dt}=\mathrm{VL} \text { and Valence_Synesketch=VL } \\
=>\mathrm{Q} 3\end{array}$ & $8.3 / 71$ \\
\hline 18 & $\begin{array}{l}\text { anger_LIWC }=\mathrm{VL} \text { and weak_GI=VL } \\
=>\mathrm{Q} 4\end{array}$ & $10 / 72$ \\
\hline 19 & $\begin{array}{l}\text { swear_LIWC=VL and \#GAZQ4=VH } \\
=>\mathrm{Q} 4\end{array}$ & $9.4 / 73$ \\
\hline 20 & \#Slang=VL and \#GAZQ2=VL => Q4 & $8.8 / 76$ \\
\hline 21 & $\mathrm{prp}=\mathrm{VL}$ and \#GAZQ2=VL => Q4 & $8.8 / 72$ \\
\hline
\end{tabular}

\subsubsection{Relations among features}

The same premises concerning outliers, false predictors and discretization were applied as in the prior section.

We have considered rules with a minimum representativity (support) of $10 \%$ and a minimum confidence measure of $95 \%$. After that all the rules were analyzed and redundant rules were removed.

The results (Table 21) show only the more representative rules and are in consonance with what we suspected after the analysis made in the last sections.

We briefly analyze the scope of the rules listed in Table 21.

(Rule 1) The feature GI_passive (words indicating a passive orientation) has, for the class $\mathrm{VH}$, almost all the songs in the quadrants 1 and 2 . The same happens for the features $v b$ (verb in base form) and prp (personal pronouns). We would say that this rule reveals an association among the features namely for positive activation.

(Rule 2) GI_intrj (includes exclamations as well as casual and slang references, words categorized "yes" and "no" such as "amen" or "nope", as well as other words like "damn" and "farewell") and GI_active (words implying an active orientation) both with values very high imply a VH value for the feature GI_iav (verbs giving an interpretative explanation of an action, such as "encourage, mislead, flatter"). This rule is predominantly true for the quadrant 2 .

Table 21. Rules from association mining.

\begin{tabular}{|l|l|c|}
\hline$\#$ & Association rules & $\begin{array}{c}\text { Support/ } \\
\text { Confidence } \\
(\%)\end{array}$ \\
\hline 1 & GI_passive=VH and vb=VH => prp=VH & $20 / 100$ \\
\hline 2 & $\begin{array}{l}\text { GI_intrj=VH and GI_active=VH => } \\
\text { GI_iav=VH }\end{array}$ & $19 / 100$ \\
\hline 3 & \#Slang=VH and GI_you=VH => prp=VH & $18 / 100$ \\
\hline 4 & $\begin{array}{l}\text { VinANEW=VL and Fear_W_Syn=VH => } \\
\text { Sadness_W_Syn=VH }\end{array}$ & $18 / 100$ \\
\hline 5 & $\begin{array}{l}\text { \#Slang=VH and FCL=VH and dav=VH } \\
\text { =>WC=VH }\end{array}$ & $18 / 100$ \\
\hline 6 & $\begin{array}{l}\text { strong=VH and GI_active=VH => } \\
\text { iav=VH }\end{array}$ & $22 / 95$ \\
\hline 7 & \#Slang=VL and prp=VL => WC=VL & $21 / 95$ \\
\hline 8 & \#Slang=VL and FCL=VL => WC=VL & $21 / 95$ \\
\hline 9 & vb=VH and GI_you=VH => prp=VH & $21 / 95$ \\
\hline 10 & \#Slang=VH and ji=VH => WC=VH & $19 / 95$ \\
\hline 11 & $\begin{array}{l}\text { VinGAZQ1Q2Q3Q4=VL and } \\
\text { Fear_W_Syn=VH => Sad- } \\
\text { ness_W_Syn=VH }\end{array}$ & $19 / 95$ \\
\hline 12 & \#Slang=VL and active=VL => strong=VL & $19 / 95$ \\
\hline 13 & FCL=VH and active=VH => iav=VH & $19 / 95$ \\
\hline & \multicolumn{2}{|l}{} \\
\hline
\end{tabular}

(Rule 3) the features \#Slang and you (pronouns indicating another person is being addressed directly) have higher values for quadrant 2 and this implicate and higher number of prp in the written style. This is typical from genres like hip-hop.

(Rule 4) Almost all the samples with a value VL for the feature VinANEW are in the quadrants 2 (more) and 3 (less). Fear_Weight_Synesketch has a VH value essentially in the quadrant 2. Sadness_Weight_Synesketch has higher values for quadrants 3 and 2, so probably this rule is applied more on songs of quadrant 2.

(Rule 5) We can see the association among the features \#Slang, FCL, dav (verbs of an action or feature of an action, such as run, walk, write, read) and WC (word count), all of them with high values and we know that this rule is more associated with the $2^{\text {nd }}$ quadrant.

(Rule 6) This rule is more associated to the quadrants 1 and 2. High values for the features strong (words implying strength), active and iav

(Rules 7 and 8) Almost all the songs with \#Slang, prp, FCL and WC equal to VL, belong to the quadrants 3 and 4 .

(Rule 9) The feature $v b$ has higher values for quadrant Q2 followed by quadrant Q1 while feature you has higher values for quadrant Q2 followed by the quadrant 3. Prp with VH values is predominantly in the quadrant 2 , so this rule is probably more associated to the quadrant 2 .

(Rule 10) These features, \#Slang, ij (number of adjectives) and WC have VH values essentially for the quadrants 1 and 2 .

(Rule 11) This rule is probably more applied in the quadrants 2 or 3, since the feature VinGAZQ1Q2Q3Q4 has predominantly lower values for quadrants 2 and 3, while Fear_Weight_ Synesketch has higher values in the same quadrants.

(Rule 12) The three features have VL values essentially for the quadrants 3 and 4 .

(Rule 13) The three features have $\mathrm{VH}$ values essentially for the quadrants 1 and 2 . 


\section{CONClusions ANd Future Work}

This paper investigates the role of lyrics in the MER process. We proposed new stylistic, structural and semantic features and a new ground truth dataset containing 180 song lyrics, manually annotated according to Russell emotion model. We used 3 classification strategies: by quadrants (4 categories), by arousal hemispheres ( 2 categories) and by valence meridian ( 2 categories). Comparing to the state of the art features (CBF - baseline), adding the other features included the novel features improved the results from $69.9 \%$ to $80.1 \%$ for quadrant categories, from $82.7 \%$ to $88.3 \%$ for arousal hemispheres and from $85.6 \%$ to $90 \%$ for valence meridian.

We conduct experiments to understand the relations between features and emotions (quadrants), not only for our new proposed features, but also for all the other features from the state of the art that we have used, namely CBF and features from known frameworks such as LIWC, GI, Synesketch and ConceptNet. This analysis show good results for some of the novel features in specific situations, such as StyBF (e.g. \#Slang and FCL), StruBF (e.g. \#Title), and SemBF in general. To the best of our knowledge, this feature analysis was absent from the state of the art and so we think this is also an interesting contribution. To understand how this relation works, we have identified interpretable rules that show the relation between features and emotions and the relations among features.

After the analysis of the best features, we concluded that some of the novel StruBF, StyBF and SemBF features are very important for quadrant's discrimination. For example \#Slang and FCL in StyBF, \#Title in StruBF and VinGAZQ2 in SemBF.

To further validate these experiments, we built a validation set comprising 771 lyrics extracted from the AllMusic platform, and validated by three volunteers. We achieved 73.6\% F-measure in the classification by quadrants.

In the future, we will continue with the proposal of new features, particularly at the stylistic and semantic level. Additionally, we plan to devise a bi-modal MER approach. To this end, we will extend our current ground truth to include audio samples of the same songs in our dataset.

Moreover, we intend to study emotion variation detection along the lyric to understand the importance of the different structures (e.g. chorus) along the lyric.

\section{ACKNOWLEDGMENT}

This work was supported by the MOODetector project (PTDC/EIA-EIA/102185/2008), financed by the Fundação para Ciência e a Tecnologia (FCT) and Programa Operacional Temático Factores de Competitividade (COMPETE) - Portugal. It was supported also by CISUC (Center for Informatics and Systems of the University of Coimbra).

\section{REFERENCES}

[1] F. Vignoli, "Digital Music Interaction concepts: a user study," Proc. of the 5th Int. Conference on Music Information Retrieval, 2004. (Conference proceedings)

[2] X. Hu and J. S. Downie, "Improving mood classification in music digital libraries by combining lyrics and audio," Proc. Tenth Ann. joint conf. on Digital libraries, pp. 159-168, 2010. (Conference proceedings)

[3] C. Y. Lu, J-S Hong and S. Cruz-Lara, "Emotion Detection in Textual Information by Semantic Role Labeling and Web Mining Techniques," Third Taiwanese-French Conf. on Information Technology, 2006.
[4] Y. Hu, X. Chen and D. Yang, "Lyric-Based Song Emotion Detection with Affective Lexicon and Fuzzy Clustering Method," Tenth Int. Society for Music Information Retrieval Conference, 2009.

[5] C. Laurier, J. Grivolla and P. Herrera, "Multimodal music mood classification using audio and lyrics," Proc. of the Int. Conf. on Machine Learning and Applications, 2008. (Conference proceedings)

[6] P. Juslin and P. Laukka, "Expression, Perception, and Induction of Musical Emotions: A Review and a Questionnaire Study of Everyday Listening," Journal of New Music Research, 33 (3), 217-238, 2004.

[7] M. Besson, F. Faita, I. Peretz, A. Bonnel and J. Requin, "Singing in the brain: Independence of lyrics and tunes," Psychological Science, 9, 1998.

[8] J. S. Downie, "The music information retrieval evaluation exchange (2005-2007): A window into music information retrieval research," Acoustical Science and Technology, vol. 29, no. 4, pp. 247-255, 2008.

[9] K. Hevner, "Experimental studies of the elements of expression in music," American Journal of Psychology, 48: 246-268, 1936.

[10] J. A. Russell, "A circumspect model of affect," Journal of Psychology and Social Psychology, vol. 39, no. 6, p. 1161, 1980.

[11] Y. Yang, Y. Lin, Y. Su and Chen H., "A regression approach to music emotion recognition," IEEE Transactions on audio, speech, and language processing, vol. 16, No. 2, pp. 448-457, 2008.

[12] X. Hu, J. Downie and A. Ehmann, "Lyric text mining in music mood classification," Proc. of the Tenth Int. Society for Music Information Retrieval Conference (ISMIR), Kobe, Japan, pages 411-416, 2009. (Conference proceedings)

[13] M. Zaanen and P. Kanters, "Automatic Mood Classification using tf*idf based on Lyrics," in J. Stephen Downie and Remco C. Veltkamp, editors, 11th International Society for Music Information and Retrieval Conference, 2010.

[14] D. Yang and W-S Lee, "Music Emotion Identification from Lyrics," Eleventh IEEE Int. Symposium of Multimedia, 2009.

[15] X. Hu, J. Downie, C. Laurier, M. Bay, and A. Ehmann, “The 2007 MIREX audio mood classification task: Lessons learned," in Proc. of the Intl. Conf. on Music Information Retrieval, Philadelphia, PA, 2008.

[16] J. A. Russell, "A circumspect model of affect," Journal of Psychology and Social Psychology, vol. 39, no. 6, p. 1161, 1980.

[17] J. A. Russell, "Core affect and the psychological construction of emotion," Psychol. Review, 110, 1, 145-172. , 2003.

[18] D. C. Montgomery, G. C. Runger and N. F. Hubele, "Engineering Statistics," Wiley, 1998.

[19] K. Krippendorff, "Content Analysis: An Introduction to its Methodology," $2^{\text {nd }}$ edition, chapter 11. Sage, Thousand Oaks, CA, 2004.

[20] F. Sebastiani, "Machine learning in automated text categorization," ACM Computing Surveys, 34(1):1-47, 2002.

[21] R. Mayer, R. Neumayer and A. Rauber, "Rhyme and Style Features for Musical Genre Categorization by Song Lyrics," Proc. of the Int. Conf. on Music Information Retrieval (ISMIR), pp. 337-342, 2008. (Conference proceedings)

[22] A. Taylor, M. Marcus and B. Santorini, "The Penn Treebank: an overview," Chapter 1, Volume 20 of the series Text, Speech and Language Technology pp 5-22, 2003.

[23] C. Whissell, "Dictionary of Affect in Language," in Plutchik and Kellerman (Eds.) Emotion: Theory, Research and Experience, vol 4, pp. 113-131, Academic Press, NY, 1989.

[24] M. M. Bradley and P. J. Lang, "Affective Norms for English Words (ANEW): Stimuli, Instruction Manual and Affective Ratings," Technical report C-1, The Center for Research in Psychophysiology, University of Florida, 1999.

[25] J. Fontaine, K. Scherer and C. Soriano, "Components of Emotional Meaning," A Sourcebook. Oxford University Press, 2013.

[26] C. Strapparava and A. Valitutti, “Wordnet-affect: an affective extension 
of wordnet," Proc. of the fourth Int. Conf. on Language Resources and Evaluation, pp. 1083-1086, Lisbon, 2004.

[27] Y. Yang, Y. Lin, H. Cheng, I. Liao, Y. Ho and H. Chen, “Toward multimodal music emotion classification," Advances in Multimedia Information Processing, PCM 2008, pages 70-79, 2008.

[28] B. Boser, I. Guyon and V. Vapnik, "A training algorithm for optimal margin classifiers," Proc. of the Fifth Ann. Workshop on Computational Learning Theory, pages 144-152, 1992. (Conference proceedings)

[29] M. Robnik-Šikonja and I. Kononenko, "Theoretical and Empirical Analysis of ReliefF and RreliefF," Machine Learning, vol. 53, no. 1-2, pp. 2369. 2003.

[30] R. Duda, P. Hart and D. Stork, “Pattern Recognition," New York, John Wiley \& Sons, Inc., 2000.

[31] D. Montgomery, G. Runger and N. Hubele, “Engineering Statistics," Wiley, 1998.

[32] S. Keerthi and C. Lin, "Asymptotic behaviors of support vector machines with Gaussian kernel”. Neural Computation, 15(7):1667-1689, 2003.

[33] Folland, G. B., "Real Analysis. Modern Techniques and their Applications," $2^{\text {nd }}$ ed. New York: John Wiley, 1999.

[34] R. Agrawal, T. Imieliński and A. Swami, "Mining association rules between sets of items in large databases," ACMSIGMOD Record, vol. 22, pp. 207-216, 1993.

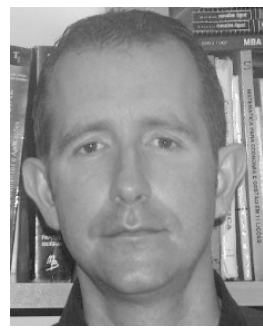

Ricardo Malheiro is PhD student at the University of Coimbra. He concluded, in the same University, his Master and Bachelor (Licenciatura - 5 years) degrees, respectively in Informatics Engineering and Mathematics (branch of Computer Graphics). He is a member of the Cognitive and Media Systems research group at the Center for Informatics and Systems of the University of Coimbra (CISUC). His main research interests and main projects are in the areas of Natural Language Processing, Detection of Emotions in Music Lyrics and Text and Text/Data Mining. He teaches at Miguel Torga Higher Institute, Department of Informatics. Currently, he is teaching Decision Support Systems, Artificial Intelligence and DataWarehouses and Big Data.

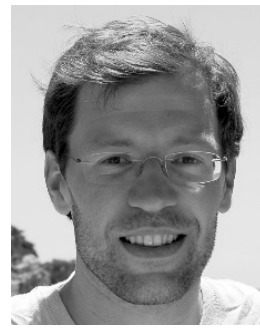

Renato Panda is a PhD student at the Department of Informatics Engineering of the University of Coimbra. He concluded his Bachelor and Master, titled "Automatic Mood Tracking in Audio Music", at the same institution. He is a member of the Cognitive and Media Systems research group at the Center for Informatics and Systems of the University of Coimbra (CISUC). His main research interests are related with Music Emotion Recognition, Music Data Mining and Music Information Retrieval (MIR). In October 2012, he was the main author of an algorithm that performed best in the MIREX 2012 Audio Train/Test: Mood Classification task, at ISMIR'2012.

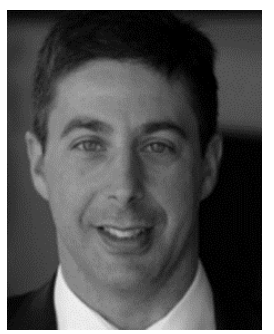

Paulo Gomes is an Assistant Professor at the Informatics Department of the University of Coimbra. He received his $\mathrm{PhD}$ from the University of Coimbra in 2004. His main research interests are: Semantic Web Technologies, Natural Language Processing, Search, Recommendation, Data/Web/Text Mining and Knowledge Management. He teaches courses like: Web Semantics, Intelligent Systems for Knowledge Management and Business Intelligence. He has directed and collaborated in more than 15 industry projects (two of them with the European Space Agency) in the areas of Knowledge Management, Information Retrieval, Semantic Search, Semantic Web Technologies, Natural Language Processing, Data Mining, Web Mining and Text Mining.

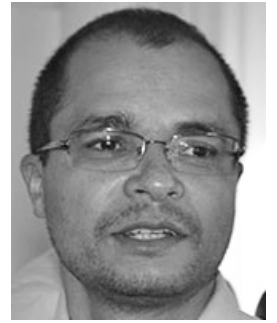

Rui Pedro Paiva is a Professor at the Department of Informatics Engineering of the University of Coimbra. He concluded his Doctoral, Master and Bachelor ( $\mathrm{Li}$ cenciatura - 5 years) degrees, all in Informatics Engineering at the University of Coimbra, in 2007, 1999 and 1996, respectively. He is a member of the Cognitive and Media Systems research group at the Center for Informatics and Systems of the University of Coimbra (CISUC). His main research interests are in the areas of Music Data Mining, Music Information Retrieval (MIR) and Audio Processing for Clinical Informatics. In 2004, Paiva's algorithm for melody detection in polyphonic audio won the ISMIR'2004 Audio Description Contest - melody extraction track, the 1st worldwide contest devoted to MIR methods. In October 2012, his team developed an algorithm that performed best in the MIREX 2012 Audio Train/Test: Mood Classification task. 